\title{
The Cyclic Butler University Game
}

\author{
Aviezri Fraenkel \\ Computer Science and Applied Mathematics \\ Weizmann Institute of Science \\ Rehovot, Israel \\ aviezri.fraenkel@weizmann.ac.il
}

April 14, 2006

\begin{abstract}
In a previous book honoring Gardner, a 2-player coin-pushing game on a directed graph (=digraph) without cycles was solved. The coins are placed on selected nodes of the "Butler University map". A move consists of choosing a coin and pushing it to an adjacent node along a directed edge. The player making the last move wins. We consider the same game, but where the digraph may be cyclic. Then there need not be a last move, in which case the outcome is a (dynamic) draw, that is, no player can force a win, but both have always a nonlosing next move. We provide an efficient strategy, consisting of deciding, for every position: (i) who can win, or (ii) whether both can only draw; and (iii) determining the next move that guarantees a win (case (i)) or maintains a draw (case (ii)).
\end{abstract}

Keywords: Cyclic games, loopy games, Generalized Sprague-Grundy function

In [4], Rebecca Wahl analyzed "The Butler University Game", created by Jerry Farrell. This 2-player game is played on a simplified map of the Butler University campus, see Fig. 1. The labels are the first letters of the various campus buildings. For example, G stands for Gallahue. Wahl explained that the roads between the buildings are directed, because a severe snowstorm led the grounds crews to mark the roads to be one-way only. Thus the map became a directed graph, also called, for short, digraph.

The game begins by placing coins on some or all of the nodes (buildings). A move consists of selecting a single coin and pushing it to an adjacent node along a directed edge, but only in the direction of the arrow. Multiple occupancy on the nodes is permitted, both in the initial placement and as the result of a move. The players move alternately. The player making the last move wins, and the opponent loses. Note that $M$ is a leaf, that is, a node without any outgoing edge. So if all coins are in $M$, play ends.

It's not immediately clear, however, that every play of the game ends in a finite number of moves. Perhaps a player can cycle through nodes, avoiding to ever enter $M$. But Fig. 2, which is a redrawing of Fig. 1, shows that the 


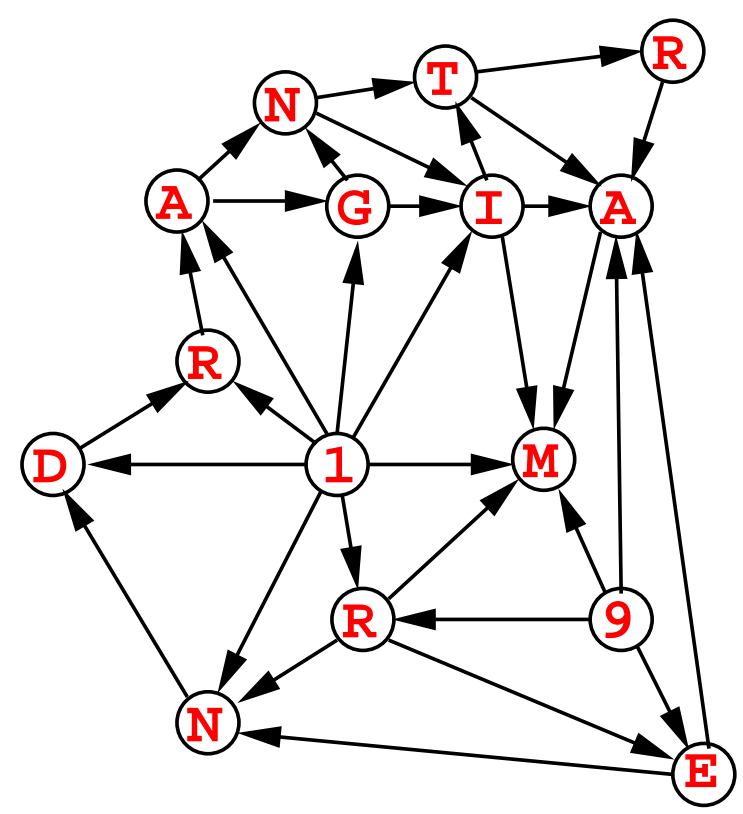

Figure 1: A simplified map of the Butler University campus.

digraph is acyclic, i.e., without cycles. So indeed every play ends with a clear winner and loser. Incidentally, Fig. 2 also shows that the Butler University paid special tribute to Martin Gardner in his 91st year of life. It is to be hoped that in at most another 9 years the University will complete its Gardner Building, so the digraph can accommodate the number 100. Using the Sprague-Grundy function, the Butler University game can be solved efficiently, i.e., a winning strategy can be computed "in polynomial time", see [1], §2.

The purpose of this note is to extend these results to cyclic digraphs digraphs that contain cycles. Then there may indeed be cases where play of the game doesn't end: no player can force a win, but each has a always a nonlosing next move. We then say that the outcome is a draw. Consider for example the case where the snow begins to thaw just a bit, so the road between $E$ and $N_{2}$ is back to 2-way traffic, see Fig. 3. If there is a single coin at $E$ or at $N_{2}$, the player venturing out of the cycle $\left(E, N_{2}\right)-\left(N_{2}, E\right)$ can be beaten by the opponent. Indeed, if Alice moves $E \rightarrow A_{1}$, then Bob moves $A_{1} \rightarrow M$, winning. If Alice moves $N_{2} \rightarrow D$, then Bob moves to $R_{2}$, Alice to $A_{2}$, and Bob chooses to move to $N_{1}$. If now Alice moves to $I$, then Bob wins immediately by going to $M$. Otherwise Alice moves to $T$. Then Bob chooses to move to $R_{1}$, Alice to $A_{1}$, Bob to $M$, winning.

Question 1. If coins are placed on some other nodes, in addition to a single coin at $E$ or $N_{2}$, does the outcome always remain a draw, or may it become a win for the first player? 


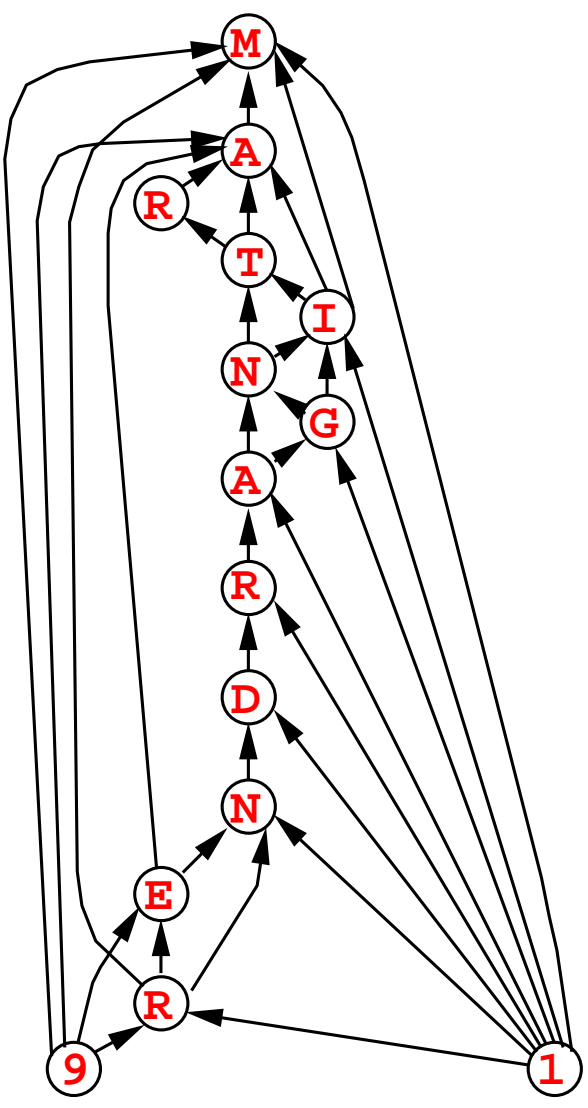

Figure 2: The snowed-in campus map is acyclic.

Every finite cyclic game can be analyzed using the Generalized SpragueGrundy function [3]. We demonstrate on the Butler University digraph how to compute this function.

Label $M$ by 0 . Do the same for every unlabeled node that doesn't have an (immediate) follower labeled 0, and every unlabeled follower of which has a follower labeled 0 . Therefore $R_{1}$ is labeled 0 . Then $N_{1}$ is labeled 0 , since the follower $I$ has the follower $M$ labeled 0 , and the follower $T$ has the follower $R_{1}$ labeled 0 . Then $R_{2}$ is labeled 0 . The next candidate is $N_{2}$ since its unlabeled follower $D$ has the follower $R_{2}$ already labeled 0 . However, it has also the unlabeled follower $E$, which doesn't have any follower labeled 0 . Thus $N_{2}$ cannot be labeled 0 . Also no other unlabeled node can be labeled 0 .

At this stage, every unlabeled node that doesn't have a follower 0 is labeled with a special symbol $\infty$, larger than every integer. Therefore we dub it infinity. This implies that $E$ and $N_{2}$ are labeled $\infty$. But the nodes $R_{3}, 1$ and 9 remain unlabeled at this stage, since each has a follower labeled 0 .

We now label by 1 every unlabeled node that doesn't have a follower 1 , and 


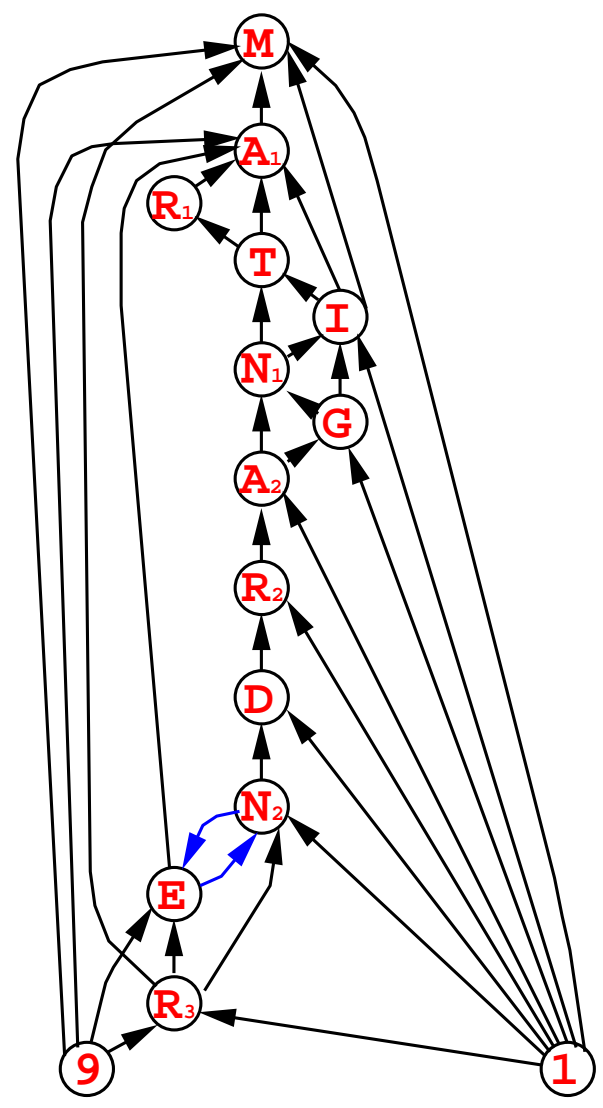

Figure 3: The snow begins to thaw.

every follower that is either unlabeled or labeled $\infty$ has a follower labeled 1. Thus $A_{1}$ is labeled 1 , then $G$ and $D$ are labeled 1 , so also $R_{3}$ is labeled 1 . Every unlabeled node has now a follower 1 , so no additional $\infty$ labels are dispensed.

Repeat this procedure with the label 2. Thus $T$ is labeled 2, then $A_{2}$. Now node 9 has the follower $\infty$ (at $E$ ) which doesn't have a follower labeled 2 , hence node 9 is labeled $\infty$. Then $I$ is labeled 3 . Node 1 is a candidate for 4 , but it has a follower $\infty$ (at $N_{2}$ ), which doesn't have a follower labeled 4 , so node 1 is labeled $\infty$. Finally, we adjoin to every $\infty$-label the set of labels of all its finite followers. The labeled digraph is depicted in Fig. 4.

We now show how to use the labels for playing the game optimally. Suppose that one coin is placed on each of the nodes $E, R_{2}$ and $I$, indicated by stars in Fig. 4. We use Nim-addition to add the labels of the occupied nodes as follows:

$$
\infty(1) \oplus 0 \oplus 3=\infty(1) \oplus 3:=\infty(1 \oplus 3)=\infty(2) .
$$

We used $\oplus$ to indicate Nim-addition, which is binary addition without carries. Thus $1 \oplus 3$ (in decimal $)=01 \oplus 11$ (in binary $)=10$ (in binary $)=2($ in decimal $)$. 


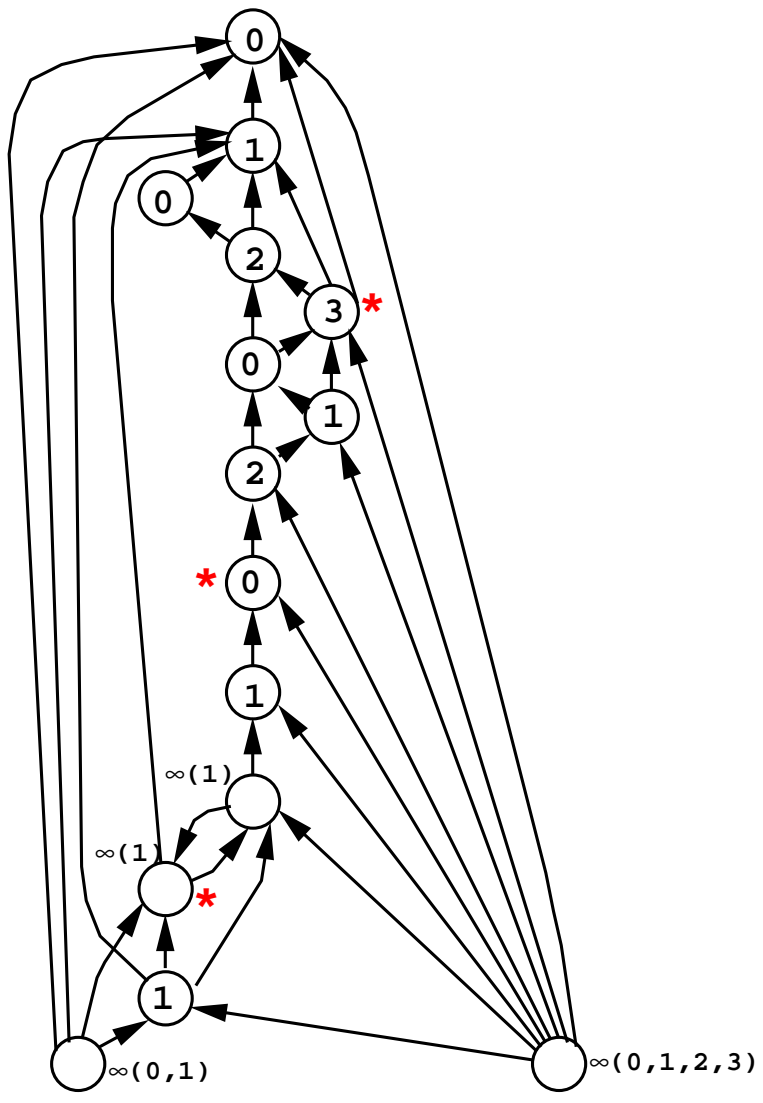

Figure 4: A fully labeled Butler University cyclic digraph.

In fact, there are only 10 types of people in the world: those who understand binary, and those who don't. Since the Nim-sum is $\infty(2)$, which doesn't contain 0 , the position is a draw. The way to maintain the draw is by moving in the cycle. If a player moves from a coin on a node labeled $\infty$ to a node labeled 1 , then the opponent can win by moving the coin on the node labeled 3 to a node labeled 1. Then the Nim sum of the resulting position is $0 \oplus 1 \oplus 1=1 \oplus 1=0$. Every position whose Nim-sum is 0 is a second-player win.

Does this indicate that the answer to Question 1, that placing coins on other nodes in addition to a coin on a node labeled $\infty$, implies that the outcome necessarily remains a draw?

Well, suppose that the initial placement of coins is as above, except that the coin on the node labeled 0 is shifted one node down, namely, it is placed initially on the node labeled 2. Then the Nim-sum of the initial position is

$$
\infty(1) \oplus 2 \oplus 3=\infty(1) \oplus 1=\infty(1 \oplus 1)=\infty(0) .
$$

Since there is a 0 attached to the $\infty$, the first player can win by moving from 
the node labeled $\infty$ to a node labeled 1 . Indeed, the resulting Nim-sum is then $1 \oplus 2 \oplus 3=0$, which is a second-player win.

In conclusion, the answer to Question 1 is that the positions of the additional coins determine whether the outcome remains a draw or becomes a win for the first player.

Now suppose that the snow has melted some more, and a loop around building $A_{2}$ (Fig. 3) became passable, in addition to the 2 -way road opened up before. Note that at $A_{2}$ there is now the option of passing: a player moving around the loop stays in the same position!

Question 2. Is it true that then every play with a coin on $A_{2}$, where there is a loop, is a draw?

Fig. 5 depicts the situation with a coin on $A_{2}$ and 2 additional coins, indi-

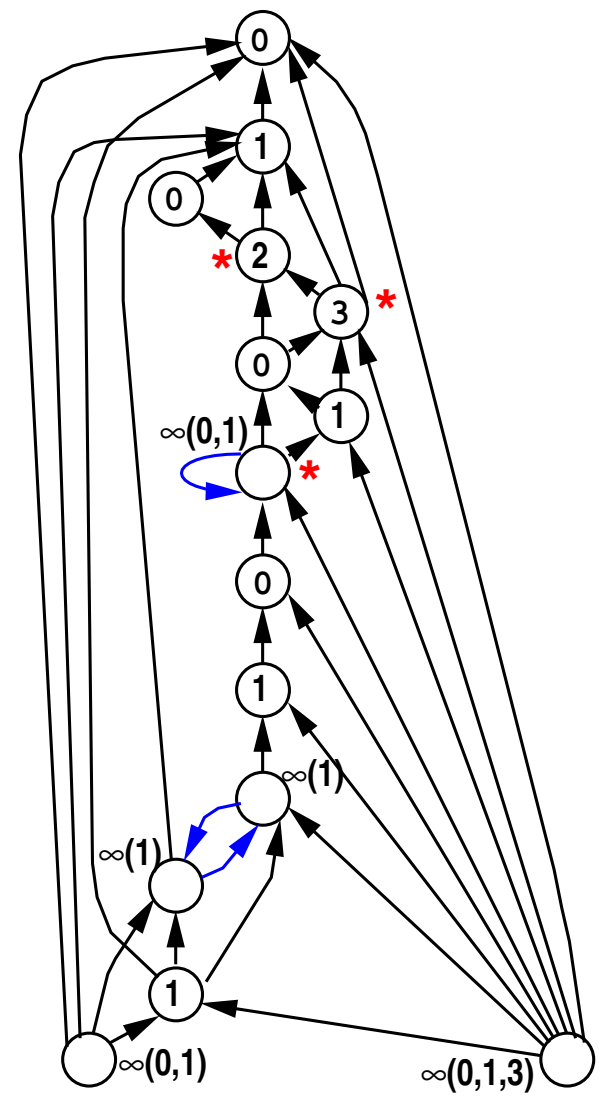

Figure 5: A fully labeled Butler University cyclic digraph, including a loop.

cated by stars, as well as the new labeling induced by the loop. The Nim-sum of the occupied nodes is now $\infty(0,1) \oplus 2 \oplus 3=\infty(0,1) \oplus 1=\infty(1,0)$. Thus the first player can win by moving to 1 . Indeed, the Nim-sum of this position 
is $1 \oplus 2 \oplus 3=0$. Note, in fact, that if there is only a single coin at $A_{2}$, then the first player can win by moving to the follower labeled 0 . However, if the initial position would have only the coin on $A_{2}$ and precisely one of the other 2 coins depicted by stars on Fig. 5 , then the result would be a draw. Thus also the answer to Question 2 is that the outcome depends on the placement of the coins.

The following is a summary of the main steps of the Generalized SpragueGrundy function labeling algorithm, for any finite digraph.

1. Put $i=0$.

2. As long as there exists an unlabeled node $u$ such that no follower of $u$ is labeled $i$ and every follower of $u$ that is either unlabeled or labeled $\infty$ has a follower labeled $i$, label $u$ by $i$.

3. Label every unlabeled node that doesn't have a follower labeled $i$ by $\infty$.

4. If there is still an unlabeled node, put $i \leftarrow i+1$ (i.e., increase $i$ by 1 ) and return to 2. Otherwise stop.

A simple short mathematical definition of the Generalized Sprague-Grundy function can be found in [1], §3. The complete labeling algorithm, also in [2], $\S 3$.

We end with a homework problem about multiple occupancy. Suppose that finitely many coins are placed on a single node $u$ of a finite digraph, all other nodes being unoccupied. Denote by $\ell(u)$ the label at $u$. Prove that:

(i) If $\ell(u)=0$, then no matter how many coins are placed on $u$, the outcome is a second player win.

(ii) If $0<\ell(u)<\infty$, then the outcome is a second player win if the number of coins on $u$ is even. Otherwise it's a first player win.

(iii) If $\ell(u)=\infty$, then the outcome is a draw for any number of coins $\geq 2$ on $u$.

\section{References}

[1] A. S. Fraenkel [1996], Scenic trails ascending from sea-level Nim to alpine chess, in: Games of No Chance, Proc. MSRI Workshop on Combinatorial Games, July, 1994, Berkeley, CA, MSRI Publ. (R. J. Nowakowski, ed.), Vol. 29, Cambridge University Press, Cambridge, pp. 13-42.

[2] A. S. Fraenkel [2000], Recent results and questions in combinatorial game complexities, Theoret. Comput. Sci. 249, 265-288.

[3] C. A. B. Smith [1966], Graphs and composite games, J. Combin. Theory 1, 51-81. 
[4] R. G. Wahl [2005], The Butler University game, in: Tribute to a Mathemagician honoring Martin Gardner (B. Cipra, E. D. Demaine, M. L. Demaine and T. Rodgers, eds.), A K Peters, Wellesley, MA, pp. 37-40. 